\title{
Ist eine Wechselwirkung zwischen dem Wetter und Kopfschmerzen nur ein Mythos?
}

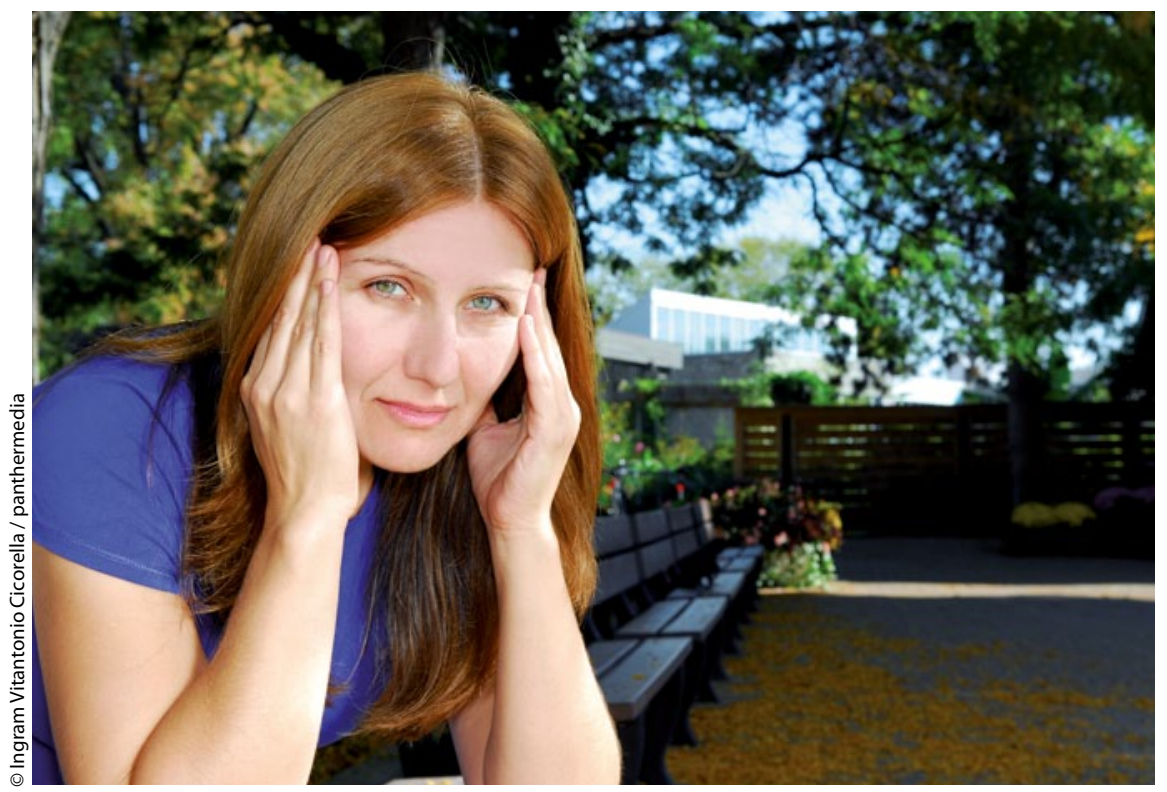

Migräne und Wetter: Wissenschaftlich besteht kein Zusammenhang, fraglich ist, ob sich die Patienten davon überzeugen lassen.

Am häufigsten nennen MigränePatienten das Wetter oder einen Wetterwechsel als Triggerfaktoren für eine Migräneattacke. In einer prospektiven Kohortenstudie wurde nun ein möglicher Zusammenhang zwischen Klimaveränderungen und Migräneanfällen untersucht.

W ährend Patienten ihre Kopfschmerzattacken häufig mit dem Wetter in Zusammenhang bringen, konnte in vielen prospektiven Studien keinerlei Zusammenhang zwischen Wetterparametern beziehungsweise Wetterwechsel und der Auslösung von Migräneattacken beobachtet werden.

Um zu untersuchen, ob ein Wetterwechsel in der Lage ist, Migräneattacken auszulösen, führten Wissenschaftler um Karin Zebenholzer von der Universitätsklinik für Neurologie in Wien eine prospektive Kohortenstudie an 238 Patienten durch, die an einer Migräne mit oder ohne Aura litten. Die Patienten mussten über 90 Tage ein dezidiertes Kopfschmerztagebuch führen. Für den Zeitraum der Studie (1. Oktober 2002 bis 31. März 2003) wurden vom Zentralinstitut für Meteorologie in Wien die täglichen Wetterdaten gemeldet. Dabei wurden elf Variablen erfasst, unter anderem Temperatur und Luftdruck. Durch eine univariate und multivariate Regressionsanalyse wurde der mögliche Zusammenhang zwischen Wetterparametern und der Auslösung von Migräne oder Kopfschmerzen berechnet. Außerdem berechneten die Forscher Korrelationen zwischen der subjektiven Wahrnehmung von Patienten bezüglich der Wettersituation und meteorologischen Daten.

Das mittlere Alter der Patienten betrug 42 Jahre, $88 \%$ waren Frauen. Im Schnitt hatten die Patienten sechs Kopfschmerzbeziehungsweise Migränetage pro Monat. Für die Analyse standen insgesamt 20.553 Patiententage zur Verfügung.

Es ergab sich für keinen der Wetterparameter ein signifikanter Zusammenhang mit der Auslösung einer Migräneattacke oder dem Auftreten von Kopfschmerzen. Im Rahmen einer Plausibilitätsanalyse wurden zusätzlich auch Parameter wie Menstruation, psychischer Stress oder Ruhephasen nach Stress erfasst. Diese Parameter zeigten einen signifikanten Zusammenhang mit dem Auftreten einer Migräne.
Lediglich für das Auftreten eines Hochdruckgebiets ergab sich ein geringer $\mathrm{Zu}$ sammenhang mit dem Auftreten von Kopfschmerzen. Die subjektive Wetterwahrnehmung korrelierte weder mit dem Auftreten noch mit dem Anhalten von Migräne oder Kopfschmerzen. Die subjektive Wahrnehmung des Wetters korrelierte relativ gut mit den Aufzeichnungen der Wetterwarte.

Schlussfolgerung: Es besteht nur ein minimaler Zusammenhang zwischen Wetterparametern beziehungsweise Wetteränderungen und dem Auftreten von Migräne oder Kopfschmerzen.

Zebenholzer $\mathrm{K}$ et al. Migraine and weather: $\mathrm{a}$ prospective diary-based analysis. Cephalalgia. 2011;31(4):391-400.

Kommentar von Prof. Diener, Essen: Diese sehr wichtige und gut durchgeführte prospektive Studie aus Österreich mit einer hohen Zahl von Patientendaten belegt, dass es praktisch keinen Zusammenhang zwischen Wetterdaten, Wetterwechsel und dem Auftreten von Migräne oder Kopfschmerzen gibt.

Die Autoren haben sich zusätzlich noch die Mühe gemacht, eine systematische Literaturrecherche zu diesem Thema durchzuführen. Sie fanden 13 Studien mit Patientenzahlen zwischen 21 und 7.054, die den Zusammenhang zwischen Wetter und Kopfschmerzen untersuchten.

Für die meisten der Studien ergab sich kein Zusammenhang zwischen Wetterparametern und dem Auftreten von Kopfschmerzen oder Migräne. Dessen ungeachtet ist es nicht zielführend, mit Migräne-Patienten Diskussionen über das Wetter als Auslöser von Kopfschmerzen zu führen, da selbst begabte Neurologen nicht in der Lage sind, das Wetter grundlegend zu beeinflussen.

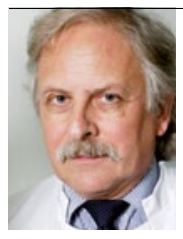

Prof. Dr. med. Hans Christoph Diener Leiter der Neurologie an der Universitätsklinik Essen 